\title{
REVIEWS OF THERAPEUTICS
}

\section{Combination Therapy with Monoamine Oxidase Inhibitors and Other Antidepressants or Stimulants: Strategies for the Management of Treatment-Resistant Depression}

\author{
Samantha J. Thomas, ${ }^{1}$ Mirae Shin, ${ }^{2}$ Melvin G. McInnis, ${ }^{3}$ and Jolene R. Bostwick ${ }^{2,4, *}$ \\ ${ }^{1}$ Meijer Pharmacy, Dewitt, Michigan; ${ }^{2}$ Pharmacy Services, University of Michigan Health System, Ann Arbor, \\ Michigan; ${ }^{3}$ Department of Psychiatry, University of Michigan Health System, Ann Arbor, Michigan; ${ }^{4}$ College of \\ Pharmacy, University of Michigan, Ann Arbor, Michigan
}

Treatment-resistant depression (TRD) is a major health concern. More than $40 \%$ of patients treated for major depressive disorder with an appropriate antidepressant dose for an adequate duration fail to respond. Further, approximately half of adults with major depressive disorder fail to achieve sustained remission despite various medication trials. The utilization of monoamine oxidase inhibitors (MAOIs) for the treatment of depression in clinical practice today is low due to their widely known adverse effects, some of which may be life threatening, and the risk for dietary and drug interactions. For these reasons, MAOIs are not recommended to be prescribed along with other antidepressants or certain prescription or nonprescription drugs. Pharmacologic options are limited for individuals with TRD, however, and there is a paucity of data on the efficacy of MAOIs in combination with other antidepressants for the management of TRD. We performed a search of the PubMed database (inception through January 25,2015$)$ to identify cases that illustrate the potential utility, as well as risks, of combination treatment with MAOIs and other antidepressants for the management of TRD; 18 articles met the criteria for our search. In addition, we performed a retrospective case series by reviewing the medical records of 29 adults treated for depression with an MAOI plus another psychotropic agent (an antidepressant or stimulant medication) between 2003 and 2012 at a large Midwestern teaching hospital. We compared the findings of the published experience with our local experience to allow for more informed decisions regarding pharmacotherapy in patients with TRD. We separated the local experience into two groups: 15 cases with the selective MAO type B inhibitor selegiline combined with medications presumed to increase the risk of serotonin syndrome and 14 cases with nonselective MAOIs (phenelzine and tranylcypromine) combined with other contraindicated medications. Although risks of combination treatment certainly exist with selective serotonin reuptake inhibitors, serotonin and norepinephrine reuptake inhibitors, or clomipramine, the current literature supports cautious use of combining MAOIs with other antidepressants in patients with TRD who have failed multiple treatment modalities. In addition, the data from the 29 patients receiving combination therapy with an MAOI and another antidepressant or stimulant medication revealed that $21 \%$ improved significantly, with no complications. This case series and literature review suggest that when used under close supervision and under the care of an experienced clinician in psychiatry, combination therapy may be a consideration for the management of TRD in patients not responding to monotherapy or other combinations of antidepressants.

KeY Words serotonin syndrome, monoamine oxidase inhibitor, MAOI, combination antidepressants. (Pharmacotherapy 2015;35(4):433-449) doi: 10.1002/phar.1576 
Treatment-resistant depression (TRD) is a major health concern. More than 40\% of patients treated for major depressive disorder (MDD) with an appropriate antidepressant dose for an adequate duration fail to respond. ${ }^{1}$ Further, approximately half of adults with MDD fail to achieve sustained remission despite various medication trials. ${ }^{2}$ Despite TRD now being a major public health concern, there is a paucity of data for the management of TRD to guide informed decision-making about pharmacotherapy. The lack of consensus on the definition of treatment resistance contributes to the deficiency of data. ${ }^{3}$ Definitions range from a poor response after proper dosing and duration of a single antidepressant to response failure after adequate dosing and duration of two or more antidepressants from different classes, ${ }^{3-5}$ which has become the working definition of TRD. ${ }^{2}$

Monoamine oxidase inhibitors (MAOIs) were one of the first classes of medications used for the treatment of depression. ${ }^{6}$ These agents inhibit the enzyme monoamine oxidase, which is present in the brain and other tissues such as the intestine and liver. ${ }^{6}$ MAOIs currently available in the United States, including selegiline, tranylcypromine, phenelzine, and isocarboxazid, irreversibly bind and inactivate monoamine oxidase, thus preventing the degradation of neurotransmitters such as serotonin, epinephrine, norepinephrine, and dopamine, leading to their accumulation. ${ }^{6}$ The use of MAOIs is limited due to their widely known adverse effects and risk for dietary and drug interactions. These include the risk of hypertensive crisis when tyraminerich foods are consumed, as well as the risk of serotonin syndrome, which can occur when MAOIs are combined with other medications, which may be life threatening. ${ }^{6,7}$ For this reason, it has historically been recommended that MAOIs

Presented as a poster at the American College of Clinical Pharmacy Virtual Poster Symposium, May 21-22, 2013; the American Society of Health-System Pharmacists Midyear Clinical Meeting, Orlando, Florida, December 8-12, 2013; and the College of Psychiatric and Neurologic Pharmacists Annual Meeting, Phoenix, Arizona, April 27-30, 2014.

*Address for correspondence: Jolene R. Bostwick, Clinical Associate Professor of Pharmacy, Department of Clinical, Social, and Administrative Sciences, College of Pharmacy, University of Michigan, and Clinical Pharmacist, Adult Psychiatry, University of Michigan Health System, 1500 E. Medical Center Drive, 9D9814 University Hospital, Ann Arbor, MI 48109-0018; e-mail: jkingsbu@med.umich. edu.

(C) 2015 Pharmacotherapy Publications, Inc. not be prescribed along with other antidepressants or certain prescription drugs (e.g., tramadol, meperidine, dextromethorphan, or methadone) and nonprescription drugs. Psychotropic agents that are contraindicated or recommended to be used with caution in combination with MAOIs are listed in Table 1. ${ }^{6,8}, 9$ In addition, a recent case study highlights the concern for severe hypertension due to the combination of high caffeine intake in combination with tranylcypromine. ${ }^{10}$ Given the severity of the risks involved with using MAOIs, in addition to the lack of marketing, fear among prescribers, and lack of experience using MAOIs, the overall use of these agents in clinical practice today is low. ${ }^{11}$

Largely, given the severity of the adverse effects and reactions, as well as relatively low utilization, a paucity of data is available on the efficacy of MAOIs in combination with other antidepressants for the management of TRD. Considering the limited pharmacologic options in individuals with TRD, additional evidence is needed to support the clinical use of combination antidepressant therapy with MAOIs and other psychotropic agents, when appropriate. Thus, in this case series and literature review, we aimed to illustrate the potential utility, as well as risks, of combination treatment in patients for the management of TRD by comparing published experience with our local experience (case series) to allow for moreinformed decisions regarding pharmacotherapy in patients with TRD.

It should be highlighted that the cases we included in the case series are separated into two groups: cases including the selective MAO type B inhibitor selegiline and cases including nonselective MAOIs (phenelzine and tranylcypromine). Selegiline confers more selective MAO-B inhibition at low doses, which improves tolerability and minimizes risk for dietary interactions with tyramine. ${ }^{12}$ However, as the dose increases, selegiline becomes less selective for MAO-B. ${ }^{13}$ A wider safety margin exists with the transdermal formulation of selegiline compared with nonselective MAOIs and oral selegiline, even in dosages up to $12 \mathrm{mg} /$ day. ${ }^{14}$ Enhanced safety with the transdermal formulation is due to avoidance of first-pass metabolism and the ability to minimize the inhibition of MAO-A in the gastrointestinal tract. ${ }^{12,14}$ Although selegiline, in any form, still confers risk in combination with other antidepressants, its selectivity should be distinguished from that of other nonselective MAOIs. 
Table 1. Psychotropic Agents Contraindicated or Recommended to Be Used with Caution in Combination with Monoamine Oxidase Inhibitors 6,8

\begin{tabular}{|c|c|c|}
\hline Psychotropic Class & Medications & Potential Reaction \\
\hline $\begin{array}{l}\text { Selective serotonin } \\
\text { reuptake inhibitors }\end{array}$ & $\begin{array}{l}\text { Fluoxetine, paroxetine, } \\
\text { sertraline, citalopram, } \\
\text { escitalopram, fluvoxamine, } \\
\text { vilazodone }\end{array}$ & Serotonin syndrome \\
\hline Tricyclic antidepressants & $\begin{array}{l}\text { Amitriptyline, imipramine, } \\
\text { clomipramine, nortriptyline, } \\
\text { amoxapine, desipramine, } \\
\text { doxepin, trimipramine, } \\
\text { protriptyline }\end{array}$ & $\begin{array}{l}\text { Serotonin syndrome, } \\
\text { hypertensive crisis }\end{array}$ \\
\hline $\begin{array}{l}\text { Serotonin and } \\
\text { norepinephrine } \\
\text { reuptake inhibitors }\end{array}$ & $\begin{array}{l}\text { Venlafaxine, duloxetine, } \\
\text { desvenlafaxine, milnacipran }\end{array}$ & Serotonin syndrome \\
\hline $\begin{array}{l}\text { Sympathomimetic } \\
\text { amines }\end{array}$ & $\begin{array}{l}\text { Amphetamines, } \\
\text { methylphenidate, } \\
\text { pseudoephedrine, } \\
\text { phenylephrine, modafinil, } \\
\text { phenylpropanolamine, } \\
\text { ephedrine, phentermine }\end{array}$ & Hypertensive crisis \\
\hline Other antidepressants & $\begin{array}{l}\text { Bupropion, mirtazapine, St. } \\
\text { John's wort, maprotiline, } \\
\text { quetiapine, lithium }\end{array}$ & $\begin{array}{l}\text { Increased risk of serious } \\
\text { adverse effects }\end{array}$ \\
\hline $\begin{array}{l}\text { Other monoamine } \\
\text { oxidase inhibitors }\end{array}$ & $\begin{array}{l}\text { Isocarboxazid, phenelzine, } \\
\text { tranylcypromine, selegiline, } \\
\text { linezolid }\end{array}$ & $\begin{array}{l}\text { Hypertensive crisis, } \\
\text { serotonin syndrome }\end{array}$ \\
\hline
\end{tabular}

\section{Methods}

\section{Literature Review}

A literature search of the PubMed database was performed. Any relevant article available in English from inception through January 25, 2015, was included. Key search terms included monoamine oxidase inhibitor, MAOI, selective serotonin reuptake inhibitor, TCA, stimulants, trazodone, antidepressants, treatment-resistant depression, depression, major depressive disorder, combination, selegiline, phenelzine, and tranylcypromine. Controlled and noncontrolled studies, open-label studies, case reports, and case series were included.

\section{Case Series}

In addition, a retrospective case series was performed by reviewing the medical records of 29 adult patients treated for depression at a large Midwestern teaching hospital; this case series was approved by the University of Michigan institutional review board. More specifically, 14 cases of a nonselective (nonselegiline) MAOI combined with other contraindicated medications and 15 cases of selegiline combined with medications presumed to increase the risk of serotonin syndrome are described. Inclusion criteria consisted of any adult patient $(\geq 18$ yrs of age) with a diagnosis of depression who was treated with an MAOI (tranylcypromine, phenelzine, selegiline, or isocarboxazid) plus another psychotropic agent, including an antidepressant or stimulant medication, between 2003 and 2012 was included. Of the 29 patients, six cases were further reviewed and are described in Data S1. These six cases were representative of the three most common combinations encountered: trazodone and an MAOI, tranylcypromine and other antidepressants, and selegiline and other antidepressants. A positive and negative clinical outcome was chosen to exemplify each combination. Information was gathered to determine safety of treatment and efficacy of combination therapy.

Medical records were reviewed and data were collected on medications used, along with dosages and durations of therapy. Duration of therapy was critical to fully determine the tolerability of therapy, given the half-lives of certain antidepressants. Any symptoms and/or adverse effects that were experienced were documented to determine tolerability to combination therapy. Patient demographics, medical and family history, summary of illness, indication for combination treatment, any necessary laboratory 
values, diet, past medication trials, social history, and allergies were also collected. Diet was evaluated based on information provided in the medical record, given that foods rich in tyramine may be the cause of any symptoms or adverse effects. Finally, use of other serotonergic agents was noted, when applicable.

\section{Results}

Eighteen articles were identified and reviewed, and are described in Table 2. ${ }^{15-32}$ Additionally, the medical records of 29 patients that mentioned the use of MAOIs in combination with other antidepressants were reviewed. These data are presented in two separate tables: Table 3 summarizes 15 cases in which the selective MAOI selegiline was used, whereas Table 4 summarizes 14 nonselegiline cases. Indications for combination therapy included depression (eight cases), TRD (six cases), recurrent MDD (nine cases), bipolar disorder (five cases), and borderline personality disorder (one case). Combinations encountered include an MAOI in combination with either trazodone (16 cases), a tricyclic antidepressant (TCA) (four cases), bupropion (two cases), an SSRI (one case), or mirtazapine (one case). Additionally, MAOIs were combined with two or more antidepressants in five cases. Of note, these patients represented a heterogeneous group, with various indications and treatment regimens.

Among the 29 patients reviewed at our institution, six patients (21\%) who received combination therapy experienced improvement in mood, with no or minimal tolerable adverse effects. Only one of these patients was receiving transdermal selegiline (Table 3, case 4), whereas four were treated with tranylcypromine (Table 4, cases 5, 8, 11, and 13) and one with phenelzine (Table 4, case 12). Similarly, seven patients (24\%) demonstrated no or minimal improvements in mood. Documented adverse effects requiring discontinuation of one or more of the medications occurred in 13 patients (45\%), including three suspected cases of serotonin syndrome, which were neither confirmed nor required acute intervention. Two patients using the selegiline patch discontinued treatment due to rash and other reasons. There were no reports of major serious consequences due to combination therapy with MAOIs. The most common adverse effects included hypotension, constipation, fatigue, dry mouth, and dizziness. The most frequent combinations included either trazodone or a TCA combined with an MAOI, sometimes with an additional psychotropic agent. The average duration of therapy for combination MAOI with trazodone was 21 months. At the time of this writing, the majority of patients receiving combination treatment with an MAOI, a TCA, and possibly another antidepressant were still taking the combination, with the exception of two cases.

\section{Discussion}

The following highlights the findings from both the literature review and our case series. There were six combinations encountered, consisting of MAOIs in combination with other antidepressants and/or stimulants.

\section{MAOI plus SSRI/SNRI}

There is a paucity of controlled, prospective data on combination therapy with an MAOI and a selective serotonin reuptake inhibitor (SSRI) or serotonin and norepinephrine reuptake inhibitor (SNRI) due to the general recommendations to avoid combination use. Combination therapy of MAOIs and SSRIs or SNRIs is dangerous due to the increased risk for serotonin syndrome. $6,33,34$ Further, deaths associated with combination use involving SSRIs have been reported in patients receiving therapeutic and overdose amounts. ${ }^{35,36}$ To avoid this, it is recommended that SSRIs or SNRIs be discontinued for at least 2 weeks before beginning MAOI therapy, with the exception of fluoxetine, which requires a 5 -week washout period. ${ }^{37}$ Our single case of fluoxetine combined with an MAOI was uninformative (see details in Table 3, case 1), as the MAOI was discontinued at an outside hospital for unknown reasons. Further, in case 1 in Table 4 and case 2 in Table 3, when the SNRIs venlafaxine and duloxetine were used in combination with an MAOI and trazodone, no positive outcomes were noted. Data support avoiding combinations of SSRIs or SNRIs with MAOIs (regardless of MAO selectivity), as the risk most often exceeds any potential benefit, particularly with concomitant use of trazodone. $6,15,16,33$

\section{MAOI plus Trazodone}

Trazodone is effective for sleep, and an openlabel study and case series have shown that low-dose trazodone may be a safe and effective treatment option for insomnia associated with 


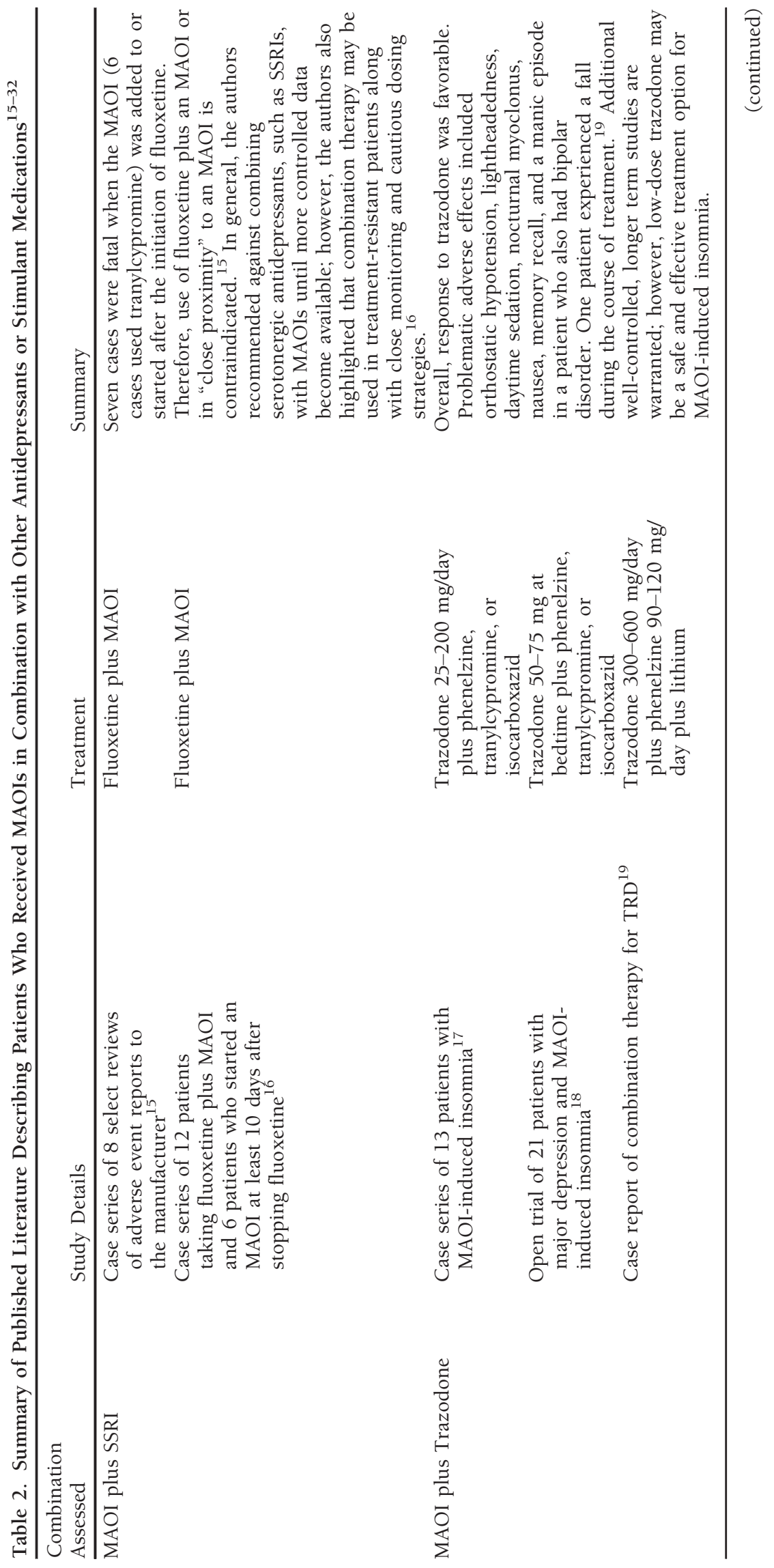




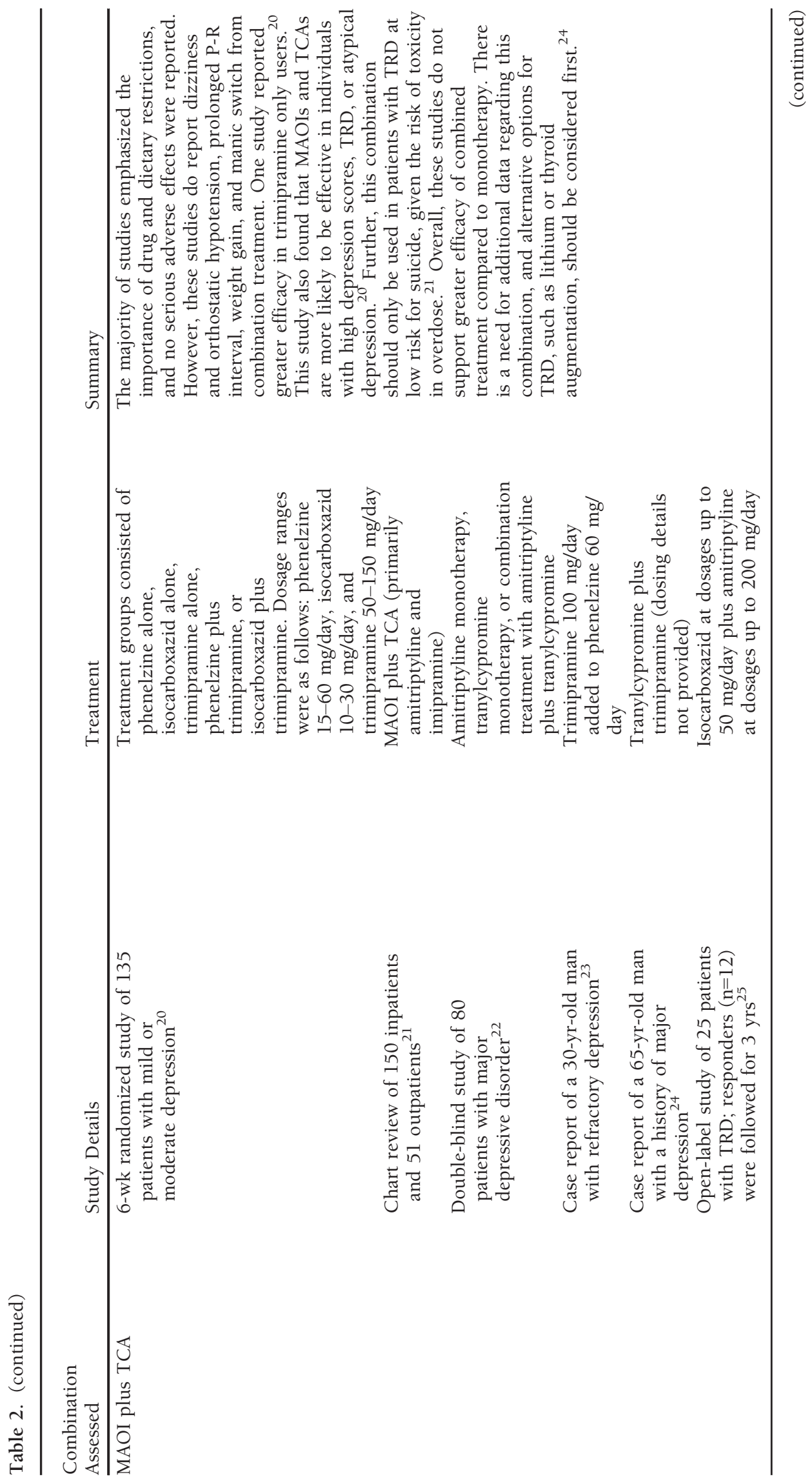




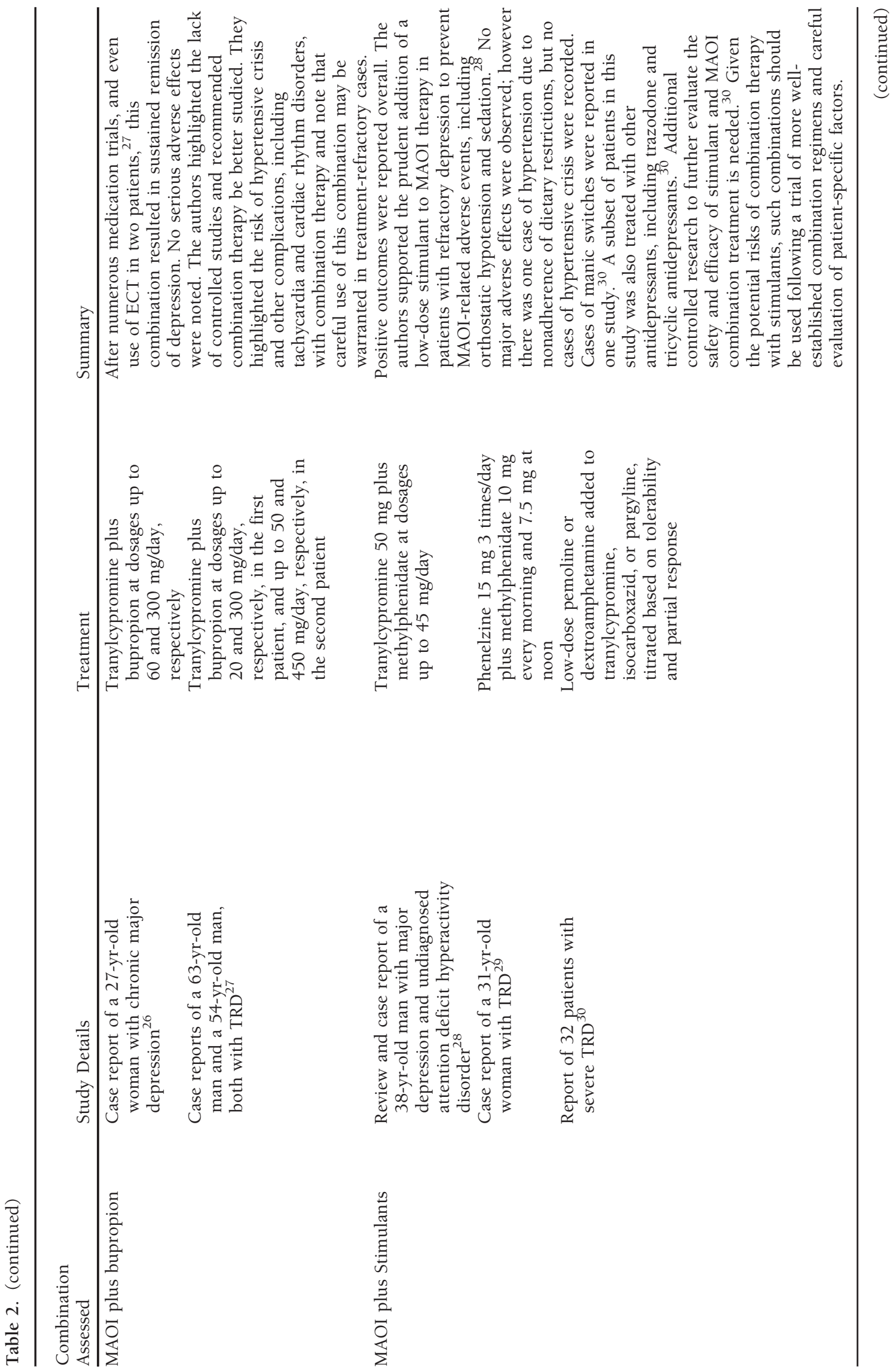




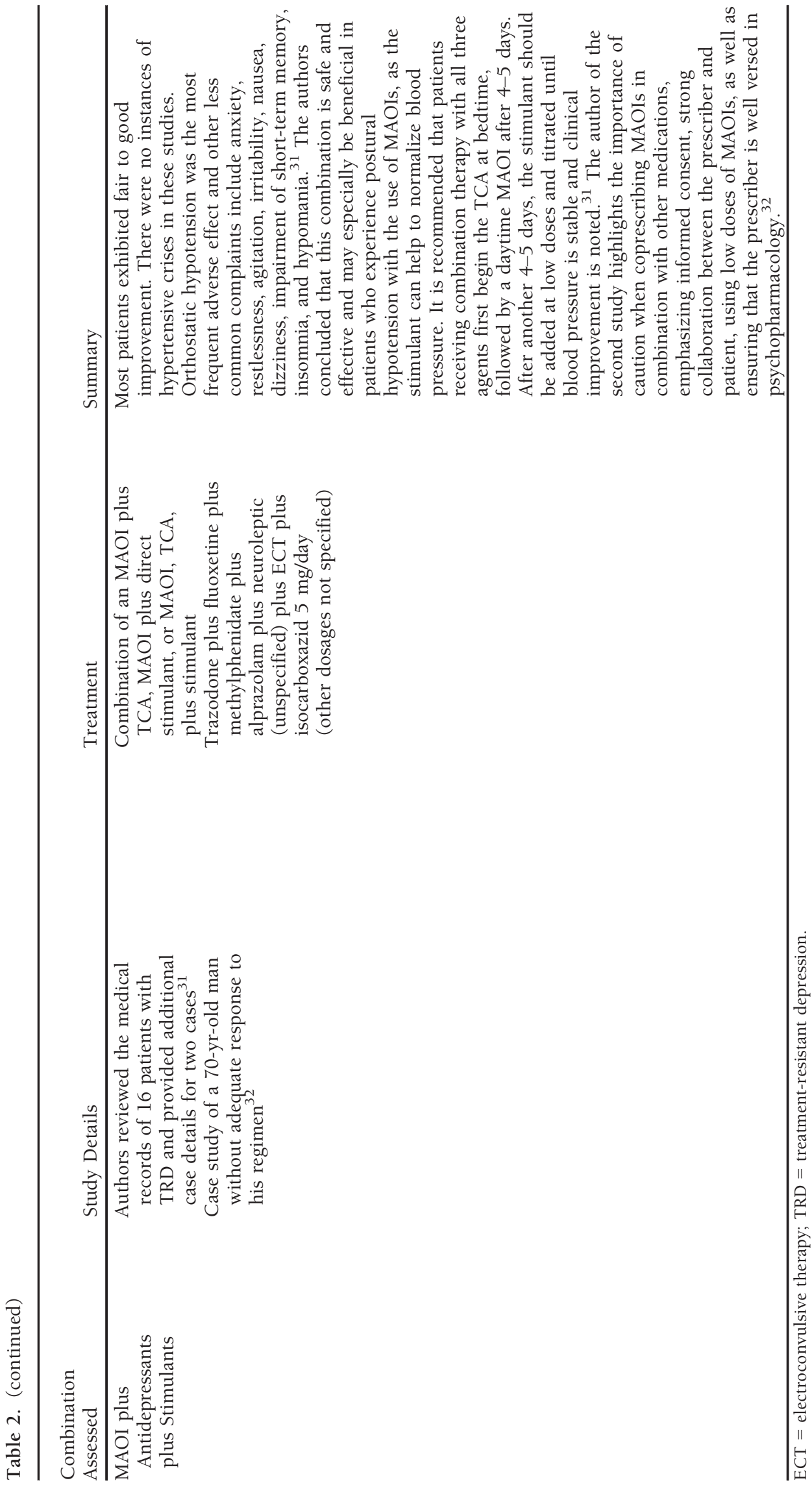




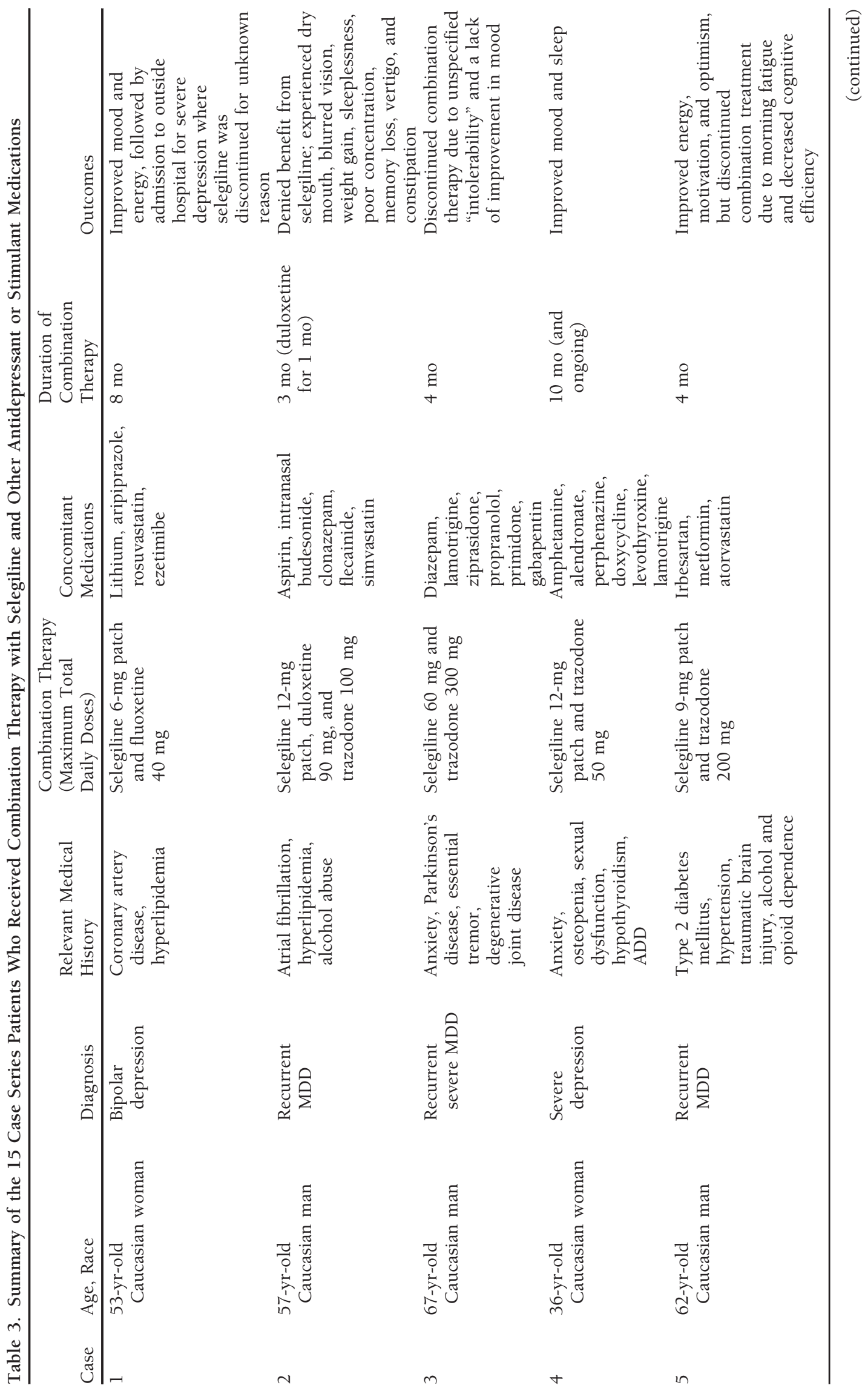




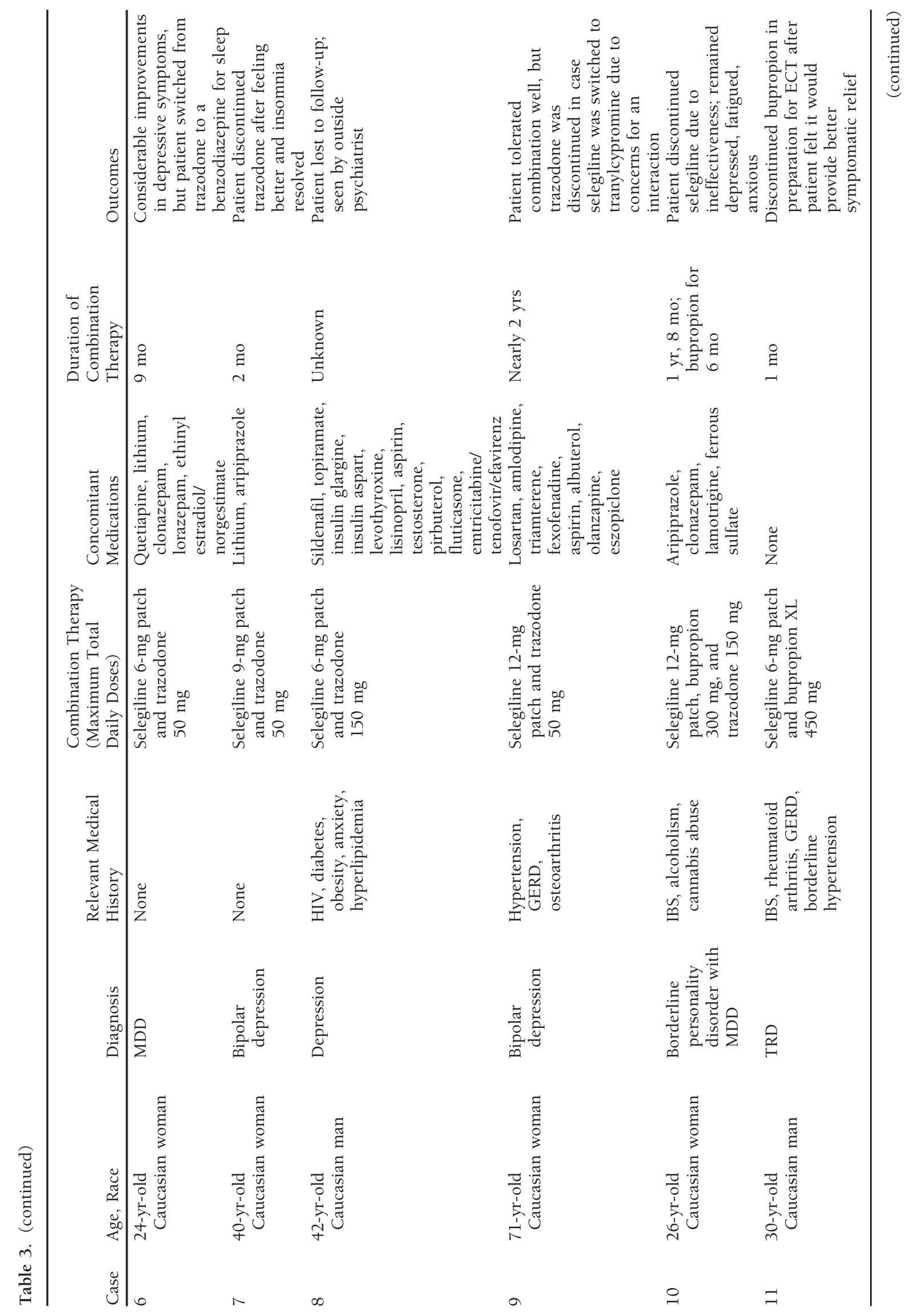




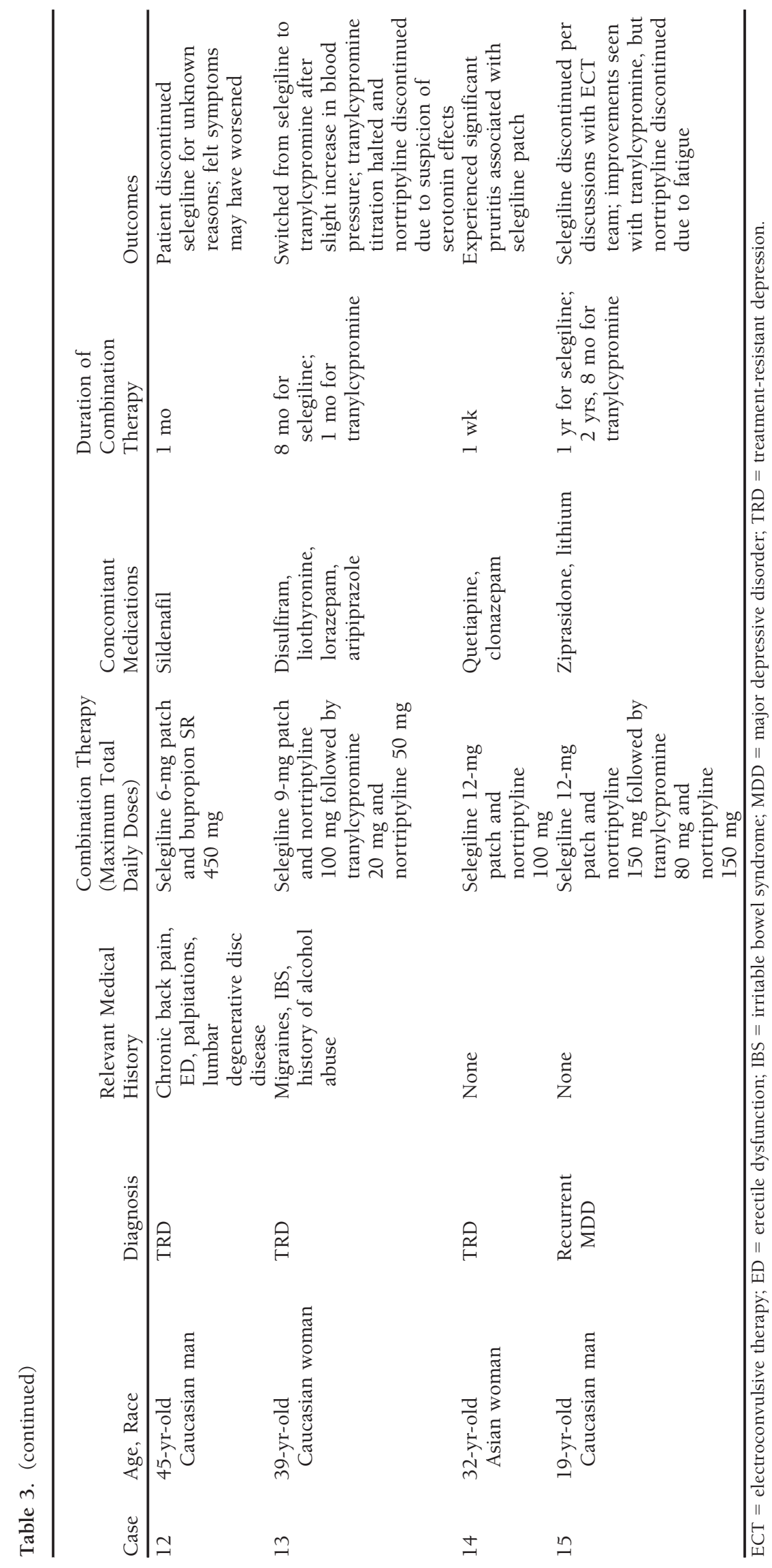


PHARMACOTHERAPY Volume 35, Number 4, 2015

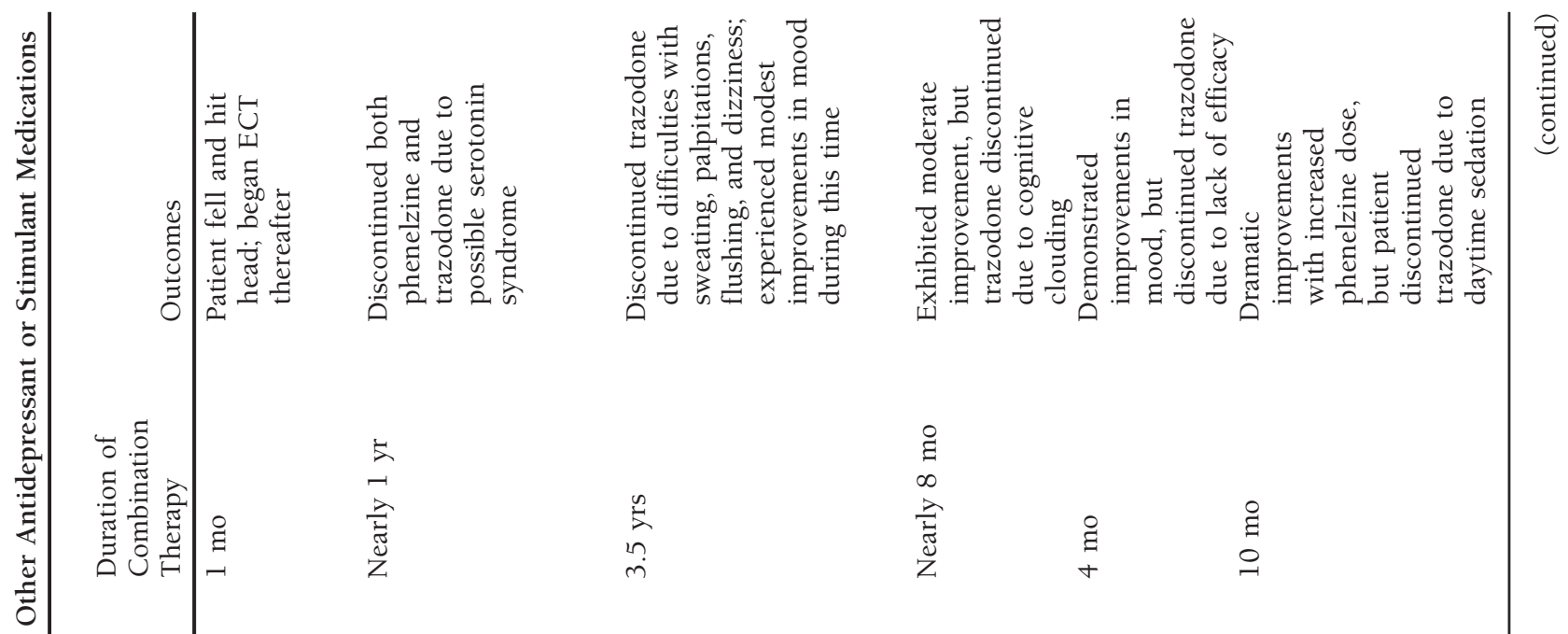

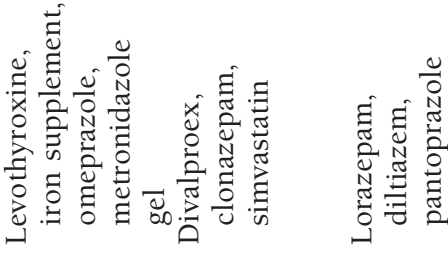

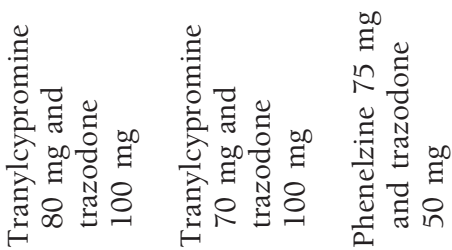

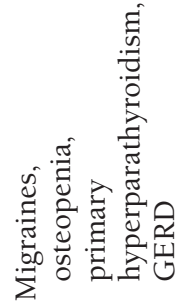




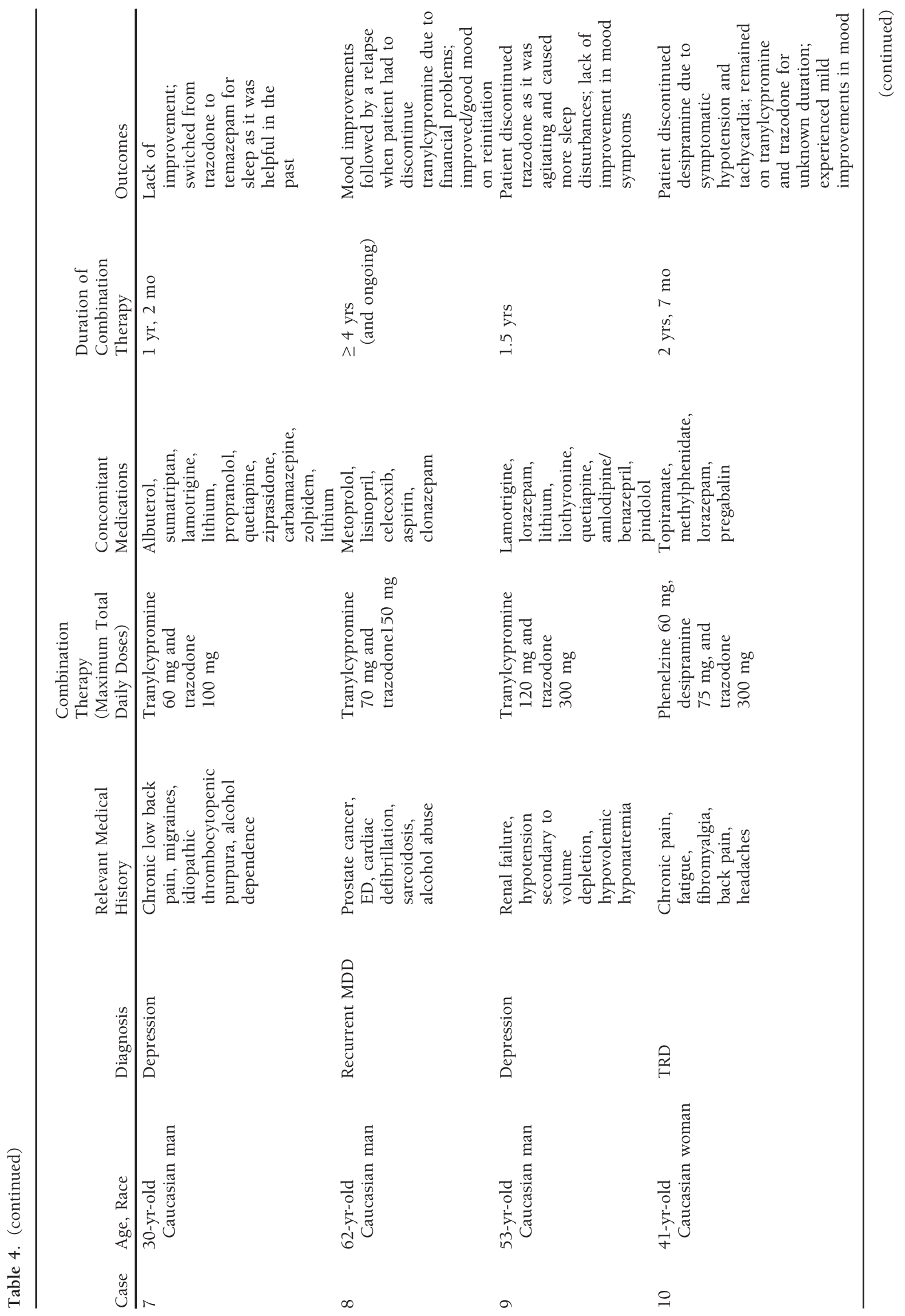




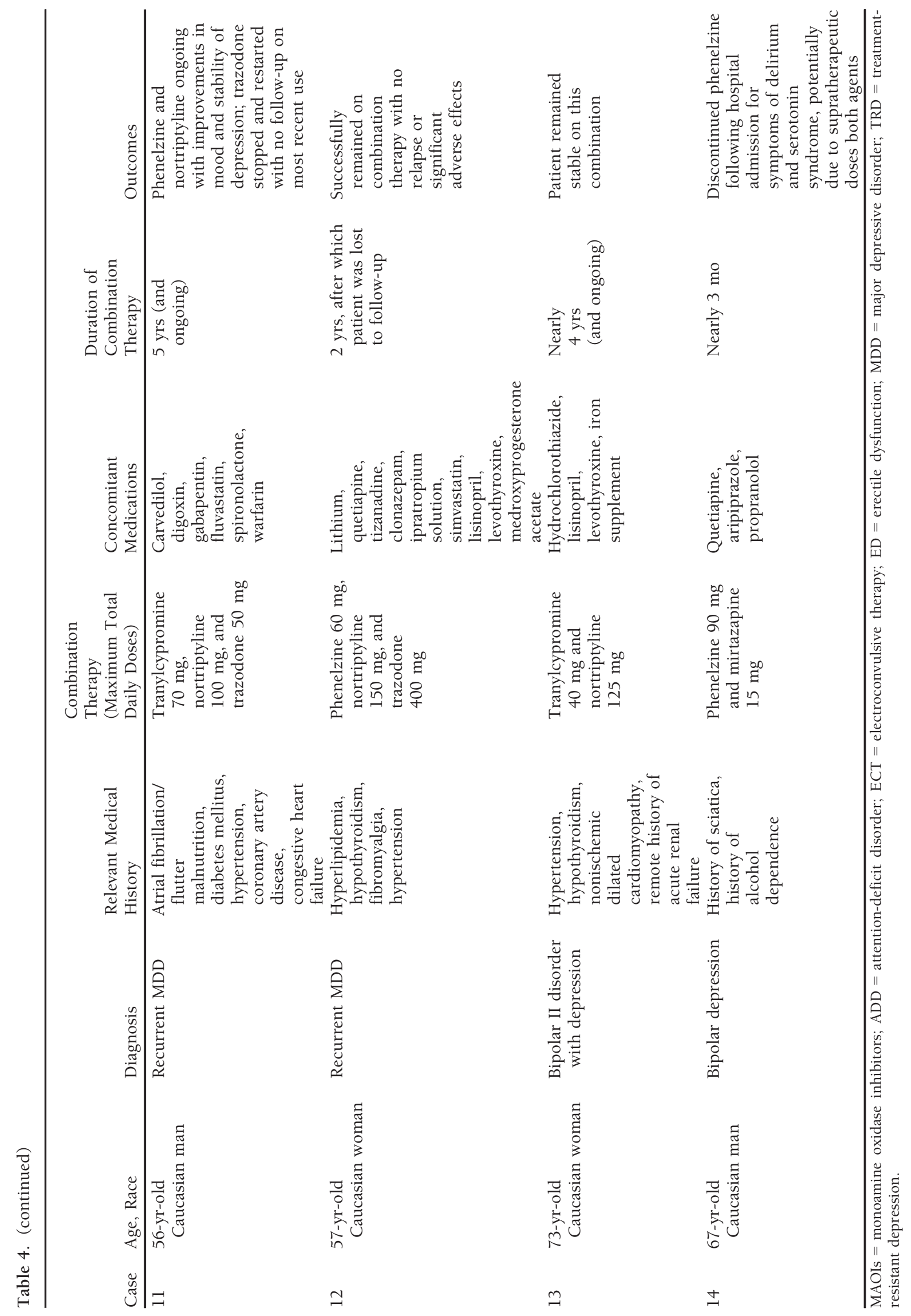


MAOI use. ${ }^{17-19}$ There are limited data on the use of higher doses of trazodone in combination with MAOIs for TRD. ${ }^{18,19}$ Overall, our cases also support safe and effective use of low-dose trazodone and MAOIs. It should be noted that the trazodone doses used are largely targeting insomnia and are not effective antidepressant doses. The addition of low-dose trazodone to MAOI therapy appeared to improve tolerability of the MAOI and overall clinical improvement of patients. Reasons for discontinuation of trazodone and MAOI combination therapy in our review included ineffectiveness, feeling overly tired, experiencing more sleep disturbance, or cognitive clouding. One patient we described in our case series stopped trazodone and MAOI combination treatment due to symptoms consistent with serotonin syndrome, although this was not definitively diagnosed (Table 4, case 2). Overall, however, patients receiving this combination averaged approximately 21 months of utilization. Further studies are needed, and the risks of combination treatment should remain a concern, including the risk of orthostatic hypotension, which should be monitored closely. It should be noted that higher doses of trazodone are likely associated with greater risk of serotonin syndrome, and the risk would likely outweigh potential benefit in most cases.

\section{MAOI plus TCA}

The literature on TCA-and-MAOI combination therapy is mixed. Small studies and case reports have demonstrated its safety, but the combination has generally been found to be less well tolerated than either agent alone. ${ }^{20-22}$ In addition, there are studies that suggest that the combination is no more efficacious than either agent alone and should be reserved for individuals with high depression scores, TRD, or atypical depression. $^{20-22}$ Our patients who received TCA and MAOI combination therapy show sustained tolerability and efficacy (Table 3, cases 13-15; Table 4, cases 10-13). Three of the seven patients remained on this regimen for at least 2 years, two of whom also received trazodone in combination with the TCA and MAOI. The combination was discontinued in four patients due to one case of hypotension (patient was also receiving trazodone), one case with suspicion of serotonin syndrome, one patient who experienced fatigue with the combination, and one patient who could not tolerate pruritis associated with the selegiline patch. These results support existing data that this combination is relatively safe, with variable efficacies. If this combination is used, the TCA should be initiated first or at the same time as the MAOI. ${ }^{9}$ It should be noted here that clomipramine, a TCA, exhibits potent inhibition of serotonin should not be used in combination with an MAOI. ${ }^{38}$

\section{MAOI plus Bupropion}

There are three existing cases published reporting on the use of bupropion and tranylcypromine in combination. ${ }^{26,27}$ Each of these cases resulted in improvement in depressive symptoms without reports of hypertension or other problematic adverse effects. Among the cases in our study, one patient continued combination treatment with bupropion and selegiline beyond 1 month, but efficacy was not well documented (Table 3, case 10). Of note, this patient was receiving transdermal selegiline, which poses a lower risk for hypertensive crisis due to its increased selectivity for MAO-B at this low dose, as previously highlighted. As such, it is not as sensitive to dietary tyramine intake as higher doses of transdermal or oral selegiline or other nonselective MAOIs. Two additional patients receiving bupropion in our case series did not demonstrate benefit with combination treatment (Table 3, cases 11-12). Given the limited published evidence, bupropion in combination with an MAOI may be considered; however, the risk for hypertension or other adverse effects should be monitored closely, and additional studies of this combination are needed.

\section{MAOI plus Stimulants}

Stimulants have been used safely in combination with MAOIs. Although stimulants are not indicated for the treatment of depression, these agents are used in clinical practice as an augmentation strategy for TRD. ${ }^{39}$ The literature, of which the majority consists of case reports and case series, supports the use of stimulants in combination with an MAOI. ${ }^{28-30}$ Stimulants may help to normalize blood pressure in those experiencing hypotension as a result of MAOI therapy. It should be noted that our case series did not highlight this combination. As with the other MAOI combinations, this should be reserved for treatmentresistant patients under the supervision of a qualified and experienced clinician, carefully assessing risk versus benefit. 
MAOI plus Antidepressants plus Stimulants

The use of direct stimulants in combination with an MAOI plus another antidepressant has demonstrated safety and efficacy in case reports. ${ }^{31,32}$ Our patients showed mixed results. One patient taking amphetamine in addition to an MAOI and another antidepressant remained stable on this combination for longer than 10 months (Table 3, case 4). Another patient taking methylphenidate in addition to an MAOI and two antidepressants discontinued one of the antidepressants due to symptomatic hypotension and tachycardia, but the rest of her regimen remained the same (Table 4 , case 10 ). The addition of stimulants to MAOI therapy has generally been avoided by physicians given the risk of hypertensive and hyperthermic crises. Blood pressure should be monitored closely as well as signs of other adverse effects. Similar to other combinations, this approach should be used with caution and under the supervision of a qualified and experienced clinician, particularly as a last resort option, including failure of electroconvulsive therapy.

\section{Limitations}

The limitations of a retrospective case series highlight recall bias and the nature of recorded clinical information. In the information we reviewed, patients may not have recollected their full diet or use of over-the-counter medications that may have contributed to adverse events. This potentially results in inaccurate records and thus adverse effects associated with diet, and medications prescribed by providers outside of our system may have been missed. As a retrospective case series, our data are strictly limited to medical records. Loss of patient follow-up could be mistaken as discontinuation of therapy, and the retrospective nature also relies on clear documentation in the medical record. Other limitations include variability among patients. Each patient presented with various depression severities, medications, formulations (patch vs oral, and selective vs nonselective MAO inhibition), and dosing regimens. These variances prevent the ability to make strong general conclusions on specific combination treatments.

\section{Conclusion}

Our case series and review of the literature suggest that when used under close supervision and under the care of an experienced clinician in psychiatry, combination therapy with MAOIs and other antidepressants (with the exception of SSRIs, SNRIs, or clomipramine) or stimulants may be a consideration for the challenging management of TRD in patients not responding to monotherapy or other combinations of antidepressants or augmentation strategies.

Two recent studies evaluated the use of the MAO type B inhibitor rasagiline in patients with Parkinson's disease in combination with various antidepressants and found no reports of serotonin toxicity. ${ }^{40,41}$ Coverage of this topic is important; however, recent literature to provide guidance on how to safely ude MAOIs for TRD in combination with other antidepressants or stimulant medications is sparse. The emerging use of electronic patient records offers the opportunity to gather therapeutic and outcomes data on larger numbers of patients. It is unlikely that clinical trials with a translational focus will be sponsored to study MAOIs and other currently used antidepressants in TRD. This is unfortunate, as longitudinal studies of individuals with TRD are needed and ideally will include embedded randomized controlled trials with combination treatment that includes MAOIs and other pharmacologic strategies. Focused environmental and pharmacologic inquiry aimed at determining why treatment resistance occurs and is sustained is needed. Our study with one health system shows that the data are accessible and that with existing data mining methods, we were able to show that one in five patients with TRD responded to a combination of an MAOI and other antidepressant. This is of major importance given that depression is so common and the finding that depression is the largest cause of disability and disability-adjusted life-years worldwide; ${ }^{42,} 43$ disability due to depression is most likely TRD.

\section{References}

1. Souery D, Amsterdam J, de Montigny C, et al. Treatment resistant depression: methodological overview and operational criteria. Eur Neuropsychopharmacol 1999;9:83-91.

2. McIntyre RS, Filteau MJ, Martin L, et al. Treatment-resistant depression: definitions, review of the evidence, and algorithmic approach. J Affect Disord 2014;156:1-7.

3. Souery D, Serretti A, Calati R, et al. Switching antidepressant class does not improve response or remission in treatmentresistant depression. J Clin Psychopharmacol 2011;31:512-6.

4. Fava M. Diagnosis and definition of treatment-resistant depression. Biol Psychiatry 2003;53:649-59.

5. The European Agency for the Evaluation of Medicinal Products. Committee for Proprietary Medicinal Products (CPMP). Note for guidance on clinical investigation of medicinal products in the treatment of depression. Available from http:// www.ema.europa.eu/docs/en_GB/document_library/Scientific_ 
guideline/2009/09/WC500003526.pdf. Updated April 25, 2002. Accessed February 18, 2014.

6. Krishnan K. Revisiting monoamine oxidase inhibitors. J Clin Psychiatry 2007;68(suppl 8):35-41.

7. Dunkley EJ, Isbister GK, Sibbritt D, Dawson AH, Whyte IM. The Hunter Serotonin Toxicity Criteria: simple and accurate diagnostic decision rules for serotonin toxicity. QJM 2003;96:635-42.

8. Somerset Pharmaceuticals, Inc. Emsam [package insert]. Morgantown, WV; 2009.

9. Grady MM, Stahl SM. Practical guide for prescribing MAOIs: debunking myths and removing barriers. CNS Spectr 2012;17:2-10.

10. van der Hoeven N, Visser I, Schene A, van den Born BJ. Severe hypertension related to caffeinated coffee and tranylcypromine: a case report. Ann Intern Med 2014;160:657-8.

11. Shulman KL, Herrmann N, Walker SE. Current place of monoamine oxidase inhibitors in the treatment of depression. CNS Drugs 2013;27:789-97.

12. Standaert DG, Roberson ED. Chapter 22. Treatment of central nervous system degenerative disorders. In: Brunton LL, Chabner BA, Knollmann BC. eds. Goodman \& Gilman's the pharmacological basis of therapeutics, 12th ed. New York, NY: McGraw-Hill; 2011. Available from: http://accesspharmacy.mhmedical.com/content.aspx ?bookid=374\&Sectionid $=41266228$. Accessed January 28, 2015.

13. Sunderland T, Mueller EA, Cohen RM, Jimerson DC, Pickar D, Murphy DL. Tyramine pressor sensitivity changes during deprenyl treatment. Psychopharmacology 1985;86:432-7.

14. Azzaro AJ, Vandenberg CM, Kemper EM, Sharoky M, Oren DA, Campbell BJ. Tyramine pressor sensitivity during treatment with the selegiline transdermal system $6 \mathrm{mg} / 24 \mathrm{~h}$ in healthy subjects. J Clin Pharmacol 2006;46:933-44.

15. Beasley CM, Masica DN, Heiligenstein JH, Wheadon DE, Zerbe RL. Possible monoamine oxidase inhibitor-serotonin uptake inhibitor interaction: fluoxetine clinical data and preclinical findings. J Clin Psychopharmacol 1993;13:312-20.

16. Feighner JP, Boyer WF, Tyler DL, Neborsky RJ. Adverse consequences of fluoxetine-MAOI combination therapy. J Clin Psychiatry 1990;51:222-5.

17. Nierenberg AA, Keck PE. Management of monoamine oxidase inhibitor-associated insomnia with trazodone. J Clin Psychopharmacol 1989;9:42-5.

18. Jacobsen FM. Low-dose trazodone as a hypnotic in patients treated with MAOIs and other psychotropics: a pilot study. J Clin Psychiatry 1990;51:298-302.

19. Zetin M. Combined use of trazodone and phenelzine in depression: case report. J Clin Psychiatry 1984;45:182-3.

20. Young JP, Lader MH, Hughes WC. Controlled trial of trimipramine, monoamine oxidase inhibitors, and combined treatment in depressed outpatients. Br Med J 1979;2:1315-7.

21. Spiker DG, Pugh DD. Combining tricyclic and monoamine oxidase inhibitor antidepressants. Arch Gen Psychiatry 1976;33:828-30.

22. O'Brien S, McKeon P, O'Regan M. The efficacy and tolerability of combined antidepressant treatment in different depressive subgroups. Br J Psychiatry 1993;162:363-8.

23. Lippmann S, Baldwin H, Manshadi M. Combined trimipramine/phenelzine treatment of depression: case report. J Clin Psychiatry 1982;43:430-1.

24. White K, Simpson G. Combined tricyclic-MAOI antidepressant treatment: a reevaluation. J Clin Psychopharmacol 1981;1:264-82.

25. Berlanga C, Ortega-Soto HA. A 3-year follow-up of a group of treatment-resistant depressed patients with a MAOI/tricyclic combination. J Affect Disord 1995;34:187-92.

26. Pierre JM, Gitlin MJ. Bupropion-tranylcypromine combination for treatment-refractor depression. J Clin Psychiatry 2000;61:450-1.
27. Quante A, Zeugmann S. Tranylcypromine and bupropion combination therapy in treatment-resistant major depression: a report of 2 cases. J Clin Psychopharmacol 2012;32:572-4.

28. Feinberg SS. Combining stimulants with monoamine oxidase inhibitors: a review of uses and one possible additional indication. J Clin Psychiatry 2004;65:1520-4.

29. Shelton Clauson A, Elliot ES, Watson BD, Treacy J. Coadministration of phenelzine and methylphenidate for treatmentresistant depression. Ann Pharmacother 2004;38:508.

30. Fawcett J, Kravitz HM, Zajecka JM, Schaff MR. CNS stimulant potentiation of monoamine oxidase inhibitors in treatmentrefractory depression. J Clin Psychopharmacol 1991;11:12732.

31. Feighner JP, Herbstein J, Damlouji N. Combined MAOI, TCA, and direct stimulant therapy of treatment-resistant depression. J Clin Psychiatry 1985;46:206-9.

32. Peterson GN. Strategies for fluoxetine-MAOI combination therapy. J Clin Psychiatry 1991;52:87-8.

33. Hodgman MJ, Martin TG, Krenzelok EP. Serotonin syndrome due to venlafaxine and maintenance tranylcypromine therapy. Hum Exp Toxicol 1997;16:14-7.

34. Brubacher JR, Hoffman RS, Lurin MJ. Serotonin syndrome from venlafaxine-tranylcypromine interaction. Vet Hum Toxicol 1996;38:358-61.

35. Keltner N, Harris CP. Serotonin syndrome: a case of fatal SSRI/MAOI interaction. Perspect Psychiatr Care 1994;30:2631.

36. Neuvonen PJ, Pohjola-Sintonen S, Tacke U, Vuori E. Five fatal cases of serotonin syndrome after moclobemide-citalopram or moclobemide-clompiramine overdoses. Lancet 1993;342:1419.

37. Rapaport MH. Dietary restrictions and drug interactions with monoamine oxidase inhibitors: the state of the art. J Clin Psychiatry 2007;68(suppl 8):42-6.

38. Pittenger $\mathrm{C}$, Bloch MH. Pharmacologic treatment of obsessivecompulsive disorder. Psychiatr Clin N Am 2014;37:375-91.

39. American Psychiatric Association. Treatment of patients with major depressive disorder, 3rd ed. Arlington, VA: American Psychiatric Association; 2010. doi: 10.1176/appi.books. 9780890423387.654001. Accessed September 23, 2014.

40. Panisset M, Chen JJ, Rhyee SH, Conner J, Methena J, the STACCATO study investigators. Serotonin toxicity association with concomitant antidepressants and rasagiline treatment: retrospective study (STACCATO). Pharmacotherapy 2014;34: 1250-8 doi: 10.1002/phar.1500

41. Smith KM, Eyal E, Weintraub D, the ADAGIO Investigators. Combined rasagiline and antidepressant use in Parkinson disease in the ADAGIO study: effects on nonmotor symptoms and tolerability. JAMA Neurol 2015;72:88-95. doi: 10.1001/jamaneurol.2014.2472.

42. World Health Organization. Depression, a hidden burden, 2012. Available from http://www.who.int/mental_health/management/depression/flyer_depression_2012.pdf. Accessed September 23, 2014.

43. Lépine JP, Briley M. The increasing burden of depression. Neuropsychiatr Dis Treat 2011;7(suppl 1):3-7.

\section{Supporting Information}

The following supporting information is available in the online version of this paper:

Data S1. Additional details for select patient cases organized by combination. 